Research Paper

\title{
The effect of combined Epidural-general Anesthesia on Hemodynamic Instability during Pheochromocytoma and Paraganglioma Surgery: A multicenter retrospective cohort study
}

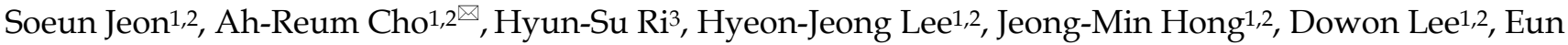 \\ Ji Park², Jinsil Kim², Christine Kang1,2 \\ 1. Department of Anesthesia and Pain Medicine, Pusan National University, School of Medicine, Yangsan, Republic of Korea. \\ 2. Department of Anesthesia and Pain Medicine, Biomedical Research Institute, Pusan National University Hospital, Busan, Republic of Korea. \\ 3. Department of Anesthesia and Pain Medicine, Pusan National University Yangsan Hospital, Yangsan, Republic of Korea. \\ $\square$ Corresponding author: Ah-Reum Cho, Mailing address: Department of Anesthesia and Pain Medicine, School of Medicine, Pusan National University, 179 \\ Gudeok-ro, Seo-gu, Busan, 49241, Republic of Korea. Tel.: +82-51-240-7399; Fax: +82-51-242-7466; E-Mail: archo@pusan.ac.kr.
}

(c) The author(s). This is an open access article distributed under the terms of the Creative Commons Attribution License (https://creativecommons.org/licenses/by/4.0/). See http://ivyspring.com/terms for full terms and conditions.

Received: 2020.02.22; Accepted: 2020.07.10; Published: 2020.07.19

\begin{abstract}
Objectives: The purpose of this study was to compare the effects of combined epidural-general anesthesia with those of general anesthesia alone on hemodynamic instability (intraoperative hypotension and hypertensive crisis) during pheochromocytoma and sympathetic paraganglioma surgery.

Methods: A total of 119 patients' medical records were reviewed who were diagnosed as having pheochromocytoma and sympathetic paraganglioma on the basis of histological findings. Intraoperative hypotension was defined as a mean blood pressure $<60 \mathrm{mmHg}$ or a decrease $>30 \%$ in baseline systolic blood pressure after adrenal vein ligation. Hypertensive crisis was defined as a systolic blood pressure $>$ $200 \mathrm{mmHg}$ or an increase $>30 \%$ in baseline systolic blood pressure during the operation. The predictor variables for intraoperative hypotension and hypertensive crisis were analyzed with logistic regression models. Data were presented as adjusted odds ratio with $95 \%$ confidence interval.

Results: The independent predictors of intraoperative hypotension were an increased attenuation number on unenhanced computed tomography (1.112 [1.009-1.226], $p=0.033)$, a high baseline mean blood pressure (1.063 [1.012-1.117], $p=0.015)$, and the combined epidural-general anesthesia $(5.439$ [1.410-20.977], $p=0.014)$. In contrast, an increased attenuation number on unenhanced computed tomography was the only independent predictor of hypertensive crisis $(1.087$ [1.021-1.158], $p=0.009)$.

Conclusions: The combined epidural-general anesthesia was not effective in attenuating hypertensive responses, but could have exacerbated intraoperative hypotension. These findings should be taken into account before selecting the anesthetic technique in pheochromocytoma and sympathetic paraganglioma surgery.
\end{abstract}

Key words: analgesia; epidural; hounsfield unit; paraganglioma; pheochromocytoma

\section{Introduction}

Pheochromocytomas and sympathetic paragangliomas (PPGLs) are chromaffin cell tumors that produce, store, and secrete catecholamines [1]. The most common symptoms of these two tumors include hypertension, headache, palpitation, and diaphoresis, all of which are caused by catecholamine excess [2].
Although the incidence of these tumors is only $0.04-0.57$ cases in 100,000 person-years [3], a diagnosis of PPGL is clinically significant. Without treatment, these tumors can lead to lethal complications, but with proper treatment, over $90 \%$ of cases are curable [4]. 
Surgical resection is the treatment of choice for PPGLs [4,5]. Recent advances in diagnostic tools, pharmacological management, and surgical and anesthetic techniques have dramatically improved the surgical outcomes of PPGLs [4,6]. However, surgical procedures for these catecholamine-secreting tumors are frequently accompanied by hemodynamic instability, which is divided into two sequential phases based on the ligation of the tumor: the hypertensive phase before tumor ligation and the hypotensive phase after tumor ligation [7]. The hypertensive crisis has been reported to occur in $51 \%-85 \%$ of PPGL surgeries [8-10]. This crisis is caused by excessive catecholamine release during endotracheal intubation, peritoneal insufflation with $\mathrm{CO}_{2}$, and tumor manipulation [11,12]. Conversely, intraoperative hypotension is known to occur in $44 \%-77 \%$ of PPGL surgeries $[9,13]$. The hypotensive event frequently occurs after tumor resection, and it is caused by intravascular volume depletion, abrupt withdrawal of catecholamine after tumor removal, and chronic downregulation of $\alpha$ and $\beta$ adrenergic receptors $[5,13,14]$. Either symptom can lead to perioperative morbidity and mortality.

The combined epidural-general anesthesia technique (GE) has been widely used in PPGL resection. This technique has been reported to facilitate hemodynamic stability before tumor isolation and reduce pain and complications after surgery [6,15]. However, the sympathectomymediated cardiovascular depression associated with an epidural block may exacerbate the intraoperative hypotension and lead to hemodynamic collapse.

The primary purpose of this retrospective cohort study was to compare the effects of GE with those of general anesthesia alone (GA) on hemodynamic instability (intraoperative hypotension and hypertensive crisis) during PPGL surgery. We also investigated the risk factors for predicting hemodynamic instability in PPGL surgery.

\section{Methods}

\section{Study design and subjects}

The institutional review boards of Pusan National University Hospital and Pusan National University Yangsan Hospital designated this retrospective cohort study as exempt (ID: H-1903-022077 and 05-2019-055). The subjects of this study were patients who had been histologically diagnosed with PPGLs from January 2000 to December 2018 at Pusan National University Hospital or Pusan National University Yangsan Hospital. The following patients were excluded from the study: those with duplicated data, patients younger than 18 years, patients with head and neck paraganglioma, patients who underwent co-operative surgery, patients who had not undergone general anesthesia, and patients with more than $10 \%$ missing values.

\section{Anesthetic management}

In the operating room, standard (electrocardiography, pulse oximetry, noninvasive blood pressure [NIBP] measurement, esophageal stethoscope temperature), depth of anesthesia (entropy or bispectral index measurement), and intraarterial blood pressure monitoring were performed. A central or large-bore peripheral intravenous catheter was inserted in all patients.

Selection of anesthetic agents and techniques was made entirely by the attending anesthesiologists. In patients who underwent GE techniques, the epidural catheter was inserted into the lower thoracic epidural space (T8-12) according to the surgical incision site prior to general anesthesia induction. To provide adequate analgesia during surgery, local anesthetics $(0.2 \%$ ropivacaine or $0.2 \%$ chirocaine) and supplemental opioid ( $3 \mathrm{mg}$ of morphine sulfate or 50 mcg of fentanyl) were epidurally administered, and intravenous remifentanil infusion was occasionally added. The administration of the epidural loading dose was completed within 30 minutes after anesthesia induction, and no epidural medication was administered during the main surgical procedure. In patients undergoing GA techniques, intravenous opioid administration (remifentanil infusion or fentanyl bolus) was used for analgesia during induction and maintenance of anesthesia.

Induction of general anesthesia was performed using an intravenous sedative hypnotic agent (thiopental sodium, propofol, or etomidate) and muscle relaxants (succinylcholine, cisatracurium, or rocuronium). Anesthesia was maintained with continuous administration of sevoflurane, desflurane, isoflurane, or propofol, along with intermittent boluses of nondepolarizing muscle relaxants (vecuronium, cisatracurium, or rocuronium).

\section{Assessment of outcomes}

Intraoperative hypotension and hypertensive crisis were defined before data collection and analysis. Intraoperative hypotension was defined as a mean intra-arterial blood pressure $<60 \mathrm{mmHg}$ or a systolic intra-arterial blood pressure (SBP) reduction $>30 \%$ immediately before induction of anesthesia $(\mathrm{SBPb})$ during the operation. Hypertensive crisis was defined by an SBP $>200 \mathrm{mmHg}$ or an increase of $>30 \%$ in the $\mathrm{SBPb}$ during the operation.

The following data were extracted from the electronic medical records: (1) baseline patient 
characteristics-age, sex, height, weight, clinical manifestations, American Society of Anesthesiologists (ASA) classification, left ventricular ejection fraction (LVEF), comorbidities; (2) preoperative computed tomography (CT) findings-The largest tumor diameter in the transverse plane and mean attenuation (Hounsfield unit: HU) on unenhanced CT. The mean attenuation number of the PPGL mass was measured using a circular region-of-interest (ROI) cursor. HU was measured in the central area while avoiding periadrenal retroperitoneal fat, and the mean HU value measured in two continuous sections was used; (3) preoperative catecholamine levelsplasma norepinephrine ( $\mathrm{pNE}$ ), plasma epinephrine (pEpi), urine vanillylmandelic acid (uVMA), and urine metanephrine (uMN) levels; (4) premedications - use of a-blocker and duration; (5) intraoperative data - anesthetic technique (GE vs. $\mathrm{GA})$, induction and maintenance agents, use of remifentanil, method of surgery (laparoscopic or open), operation time, estimated blood loss, amount of fluid administration, urine output, and blood transfusion; (6) hemodynamic data-mean blood pressure before premedication (MBPpre), mean blood pressure immediately before induction of anesthesia $(\mathrm{MBPb})$, and hemodynamic values during general anesthesia. Variables with data missing for more than $30 \%$ of the patients (plasma metanephrine, plasma normetanephrine, urine norepinephrine, urine epinephrine, and urine normetanephrine) were excluded from the analysis.

\section{Statistical analysis}

All analyses were performed using IBM SPSS Statistics (version 22; IBM Corporation, Armonk, NY) and MedCalc (version 18.11.6; MedCalc Software bvba, Ostend, Belgium). Continuous variables were presented as mean \pm standard deviation (SD) or as median and interquartile range (IQR). Categorical data were reported as absolute numbers and percentages. The results of binary logistic regression analyses were presented as odds ratio (OR) or adjusted OR with $95 \%$ confidence interval (95\% CI).

Patient data were grouped according to anesthetic technique (GE vs. GA) and the occurrence of hemodynamic instability to compare preoperative patient demographics, tumor characteristics, and intraoperative variables. After the normality test, normally distributed data were analyzed using an independent $\mathrm{t}$-test and non-parametric data were analyzed using the Mann-Whitney U test; categorical data were analyzed using the chi-squared test (with Yates' continuity correction for the $2 \times 2$ contingency table) or Fisher's exact test.

First, univariate binary logistic regression analysis was performed to evaluate the association between predictor variables and outcome variables (intraoperative hypotension and hypertensive crisis). The predictor variables entered in the univariate analysis were baseline patient characteristics, preoperative $\mathrm{CT}$ findings, preoperative catecholamine level, premedications, intraoperative data, and preoperative hemodynamic data (MBPpre and $\mathrm{MBPb}$ ).

The candidate predictors entered in the multivariate binary logistic regression analysis were predictor variables with $p$ values less than 0.25 in the univariate analysis. The anesthetic technique (GE vs. GA) was also entered in the multivariate binary logistic regression analysis regardless of $p$ value because it was our primary predictor variable. Predictor variable selection was performed by the backward elimination method based on the probability of the likelihood-ratio statistic, with $p$ value $\geq 0.1$ as the removal criterion. Patients with missing data for one or more predictors were excluded from the logistic regression analysis. To evaluate the usefulness and goodness-of-fit of the multivariate logistic regression model, Nagelkerke $\mathrm{R}^{2}$ and the Hosmer-Lemeshow chi-squared statistics were calculated. The probability of each case was calculated and the area under the receiver operating characteristic curve (AUROC) was determined to evaluate the discriminative power of the multivariate logistic regression models; AUROC was also used to determine the optimal cut-off values for continuous predictor variables.

\section{Results}

Of the 119 patient records retrieved, 15 were excluded for the following reasons: duplication, three cases; patient age under 18 years, three cases; co-operative surgery, four cases; procedures without general anesthesia, one case; and missing values, four cases (Figure 1). Of the 104 patients included in the final sample, $53(51.0 \%)$ underwent surgery with the GE technique, while the remaining 51 patients (49.0\%) underwent surgery with the GA technique. The two groups were comparable in terms of preoperative patient demographics, tumor characteristics, and intraoperative variables. In addition, the two groups did not differ in terms of preoperative hemodynamic parameters (Table 1 ).

\section{Predictors of intraopertive hypotension}

Preoperative patient demographics, tumor characteristics, and intraoperative variables in the groups stratified according to the presence of hypotension after PPGL resection are summarized in Table 2. Of all 104 patients included in this study, 
intraoperative hypotension occurred in 80 (76.9\%) patients. There were significant differences in the baseline BP and anesthetic technique between the two groups.

Table 1. Patient demographics, tumor characteristics, and intraoperative variables according to anesthetic technique

\begin{tabular}{|c|c|c|c|}
\hline \multirow[t]{2}{*}{ Variables } & \multicolumn{2}{|l|}{ Anesthetic techniques } & \multirow[t]{2}{*}{ P value } \\
\hline & GA $(n=51)$ & GE $(n=53)$ & \\
\hline Age (yr) & $49.9(14.8)$ & $48.8(13.0)$ & 0.685 \\
\hline Sex (Male) & $26(51.0)$ & $30(56.6)$ & 0.705 \\
\hline ASA classification & & & 0.297 \\
\hline I & $7(13.7)$ & $5(9.4)$ & \\
\hline II & $36(70.6)$ & $44(83.0)$ & \\
\hline III & $8(15.7)$ & $4(7.5)$ & \\
\hline Height (cm) & $163.8(8.6)$ & $164.5(7.9)$ & 0.694 \\
\hline Weight (kg) & $62.0(9.8)$ & $63.5(12.1)$ & 0.487 \\
\hline BMI $\left(\mathbf{k g} / \mathbf{m}^{2}\right)$ & $23.0(3.0)$ & $23.3(3.5)$ & 0.628 \\
\hline Preoperative LVEF (\%) & $63.0(60.0-65.5)$ & $64.0(60.0-65.5)$ & 0.694 \\
\hline Clinical manifestations & $31(60.8)$ & $37(69.8)$ & 0.447 \\
\hline \multicolumn{4}{|l|}{ Comorbidity } \\
\hline Stroke & $5(9.8)$ & $1(1.9)$ & 0.109 \\
\hline Dysarrhythmia & $6(11.8)$ & $2(3.8)$ & 0.157 \\
\hline Hyperlipidemia & $2(3.9)$ & $3(5.7)$ & 1.000 \\
\hline Diabetes mellitus & $14(27.5)$ & $20(37.7)$ & 0.364 \\
\hline \multicolumn{4}{|l|}{ Preoperative CT findings } \\
\hline HU on unenhanced CT & $33.9(7.4)$ & $34.0(9.2)$ & 0.963 \\
\hline Tumor diameter $(\mathrm{cm})$ & $6.0(3.6-36.0)$ & $5.3(3.6-8.0)$ & 0.474 \\
\hline \multicolumn{4}{|c|}{ Preoperative catecholamine levels } \\
\hline $\begin{array}{l}\text { Plasma norepinephrine } \\
(\mathrm{pg} / \mathrm{ml})\end{array}$ & $343.2(82.0-947.6)$ & $342.0(11.5-878.8)$ & 0.872 \\
\hline Plasma epinephrine $(\mathrm{pg} / \mathrm{ml})$ & $31.8(0.5-176.3)$ & $72.4(6.1-151.3)$ & 0.716 \\
\hline $\begin{array}{l}\text { Urine vanillylmandelic acid } \\
\text { (mg/day) }\end{array}$ & $10.0(6.0-20.9)$ & $9.1(6.0-17.5)$ & 0.523 \\
\hline $\begin{array}{l}\text { Urine metanephrine } \\
(\mu \mathrm{g} / \text { day })\end{array}$ & $2029.0(901.5-6993.5)$ & $3700.0(353.7-3700.0)$ & 0.250 \\
\hline \multicolumn{4}{|l|}{ Preoperative MBP (mmHg) } \\
\hline Before premedication & $98.5(93.3-111.2)$ & $100.0(92.8-110.0)$ & 0.829 \\
\hline $\begin{array}{l}\text { Before induction of } \\
\text { anesthesia }\end{array}$ & $96.7(86.7-109.7)$ & $102.0(90.0-114.7)$ & 0.366 \\
\hline \multicolumn{4}{|l|}{ Premedication } \\
\hline a-blocker & $46(90.2)$ & $47(88.7)$ & 1.000 \\
\hline $\begin{array}{l}\text { Duration of pretreatment } \\
\text { (days) }\end{array}$ & $30.0(15.0-43.0)$ & $28.0(20.3-45.8)$ & 0.966 \\
\hline \multicolumn{4}{|l|}{ Intraoperative data } \\
\hline Induction agent & & & 0.602 \\
\hline Thiopental & $15(29.4)$ & $18(34.0)$ & \\
\hline Propofol & $36(70.6)$ & $34(64.2)$ & \\
\hline Etomidate & $0(0.0)$ & $1(1.9)$ & \\
\hline Maintenance agent & & & 0.614 \\
\hline Inhalation & $49(96.1)$ & $52(98.1)$ & \\
\hline Propofol & $2(3.9)$ & $1(1.9)$ & \\
\hline Remifentanil & $47(92.2)$ & $30(56.6)$ & $<0.001$ \\
\hline Methods of surgery & & & 0.324 \\
\hline Open & $11(21.6)$ & $17(32.1)$ & \\
\hline Laparoscopic & $40(78.4)$ & $36(67.9)$ & \\
\hline Intraoperative hypotension & $33(64.7)$ & $47(88.7)$ & 0.008 \\
\hline Hypertensive crisis & $30(58.8)$ & $27(50.9)$ & 0.542 \\
\hline
\end{tabular}

Data were presented as mean (SD), median (IOR), and absolute numbers (\%). Combined epidural-general anesthesia technique (GE), general anesthesia alone (GA), American society of anesthesiologists (ASA), body mass index (BMI), left ventricular ejection fraction (LVEF), computed tomography (CT), Hounsfield unit (HU), mean blood pressure (MBP)

Univariate analysis presented 6 candidate predictors that might be independently associated with the occurrence of intraoperative hypotension (Table 3). The following six candidate predictors were entered in the multivariate logistic regression analysis: $\mathrm{HU}$ value on unenhanced $\mathrm{CT}$, MBPpre, $\mathrm{MBPb}$, a-blocker premedication, duration of pretreatment, and anesthetic technique. The results of variable selection using the backward elimination method showed that the following variables were independent predictors of intraoperative hypotension: increased HU value on unenhanced CT (adjusted OR [95\% CI]: 1.112 [1.009-1.226], $\mathrm{p}=0.033$ ), high $\mathrm{MBPb}$ (adjusted OR [95\% CI]: 1.063 [1.012-1.117], $\mathrm{p}=0.015$ ), and GE technique (adjusted OR [95\% CI]: 5.439 [1.410-20.977], $\mathrm{p}=0.014$ ). a-Blocker premedication was included in the final model, but it was not statistically significant (adjusted OR [95\% CI]: 5.459 [0.937-31.805], $\mathrm{p}=0.059 ;$ Table 4). The Hosmer-Lemeshow chi-square statistic, Nagelkerke $\mathrm{R}^{2}$, and AUROC of the final model were $4.957(\mathrm{p}=$ $0.762), 0.403$, and 0.852 (95\% CI: 0.765-0.938), respectively. The receiver operating characteristic (ROC) analysis to determine the cut-off values of continuous predictor variables showed that the optimal cut-off HU value on unenhanced CT and $\mathrm{MBPb}$ were $37 \mathrm{HU}$ (AUROC [95\% CI]: 0.654 [0.539-0.757]) and $93.7 \mathrm{mmHg}$ (AUROC [95\% CI]: 0.741 [0.646-0.822]).

\section{Predictors of hypertensive crisis}

Preoperative patient demographics, tumor characteristics, and intraoperative variables of the groups stratified according to the occurrence of hypertensive crisis during PPGL resection are summarized in Table 2; hypertensive crises occurred in $57(54.8 \%)$ patients. There were significant differences in age, $\mathrm{HU}$ value on unenhanced $\mathrm{CT}$, and tumor diameter between the two groups.

Seven candidate predictors (Table 3) and anesthetic technique were entered in the multivariate logistic regression analysis. The results of variable selection using the backward elimination method showed that an increased HU value on unenhanced CT was the only independent predictor for the occurrence of hypertensive crisis (adjusted OR [95\% $\mathrm{CI}$ : 1.087 [1.021-1.158], $\mathrm{p}=0.009$ ). Dysarrhythmia (adjusted OR [95\% CI]: 0.026 [0.032-1.318], $\mathrm{p}=0.095$ ) and GE technique (adjusted OR [95\% CI]: 0.378 [0.141-1.015], $\mathrm{p}=0.053$ ) were included in the final model, but were not statistically significant (Table 4). The Hosmer-Lemeshow chi-squared statistic, Nagelkerke $\mathrm{R}^{2}$, and AUROC of the final model were $6.010(\mathrm{p}=0.646), 0.198$, and 0.727 (95\% CI: 0.616, $0.838)$, respectively. The ROC analysis to determine the cut-off value of the continuous predictor variable showed that the optimal cut-off $\mathrm{HU}$ value on unenhanced CT was 36 HU (AUROC [95\% CI]: 0.667 [0.553-0.768]). 


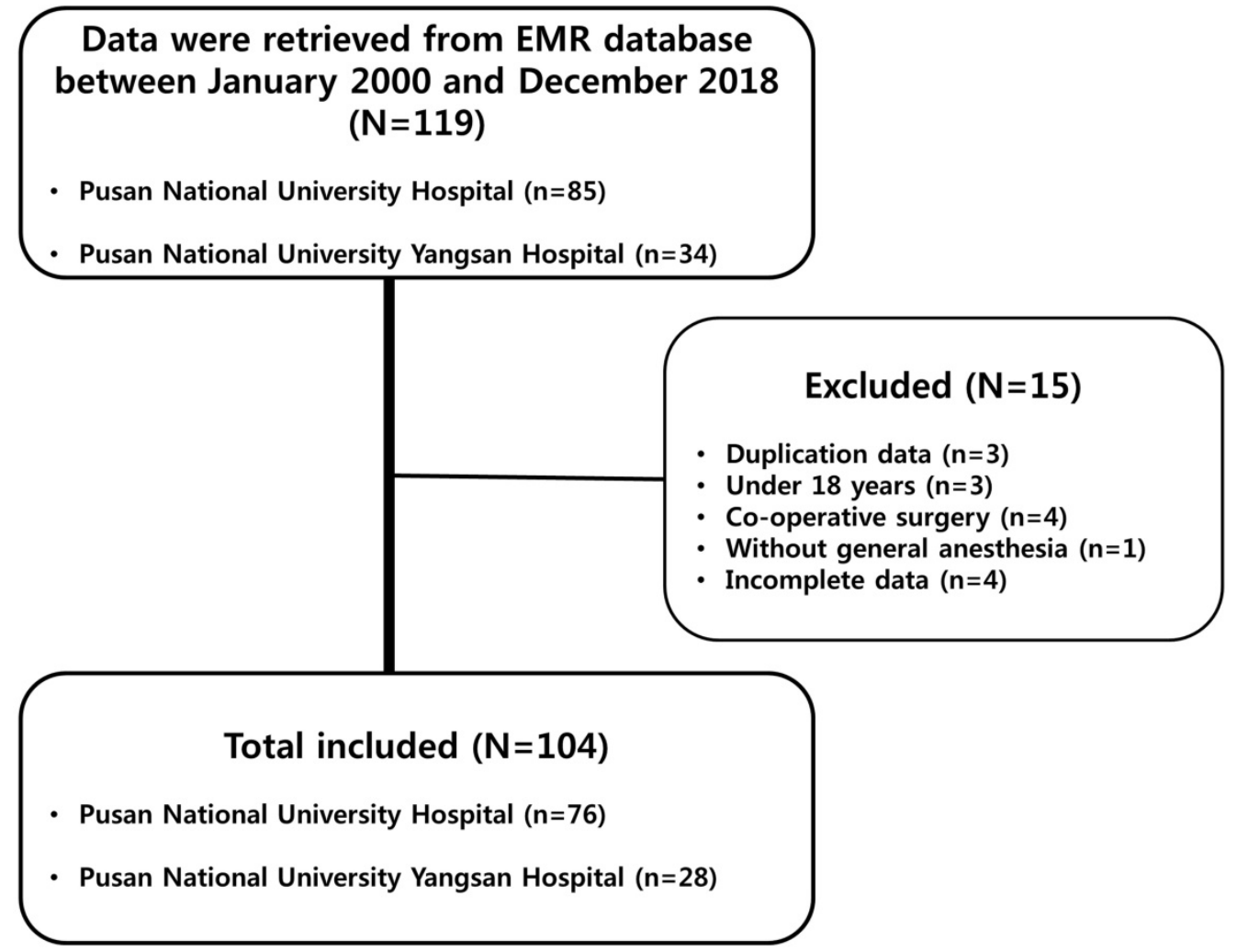

Figure 1. Study flow chart. Of the 119 patients' records retrieved, 15 were excluded for the following reasons: 3 due to duplication, 3 due to patients' age under 18 years old, 4 due to patients underwent co-operative surgery, 1 due to procedures without general anesthesia, and 4 due to missing values. Electronic medical record (EMR).

Table 2. Patient demographics, tumor characteristics, and intraoperative variables according to the occurrence of intraoperative hypotension and hypertensive crisis

\begin{tabular}{|c|c|c|c|c|c|c|}
\hline \multirow[t]{2}{*}{ Variables } & \multicolumn{3}{|c|}{ Intraoperative hypotension } & \multicolumn{3}{|l|}{ Hypertensive crisis } \\
\hline & No. $(\mathrm{n}=24)$ & Yes $(n=80)$ & P value & No. $(\mathrm{n}=47)$ & Yes $(\mathrm{n}=57)$ & P value \\
\hline Age (yr) & $49.3(12.1)$ & $49.3(14.4)$ & 0.998 & $52.7(13.3)$ & $46.5(13.8)$ & 0.022 \\
\hline Sex (Male) & $13(54.2)$ & $43(53.8)$ & 1.000 & $25(53.2)$ & $31(54.4)$ & 1.000 \\
\hline ASA classification & & & 0.149 & & & 0.385 \\
\hline I & $5(20.8)$ & $7(8.8)$ & & $3(6.4)$ & $9(15.8)$ & \\
\hline II & $15(62.5)$ & $65(81.3)$ & & $38(30.9)$ & $42(73.7)$ & \\
\hline III & $4(16.7)$ & $8(10.0)$ & & $6(12.8)$ & $6(10.5)$ & \\
\hline Height (cm) & $163.6(5.9)$ & $164.3(8.8)$ & 0.655 & $163.5(9.0)$ & $164.7(7.6)$ & 0.464 \\
\hline Weight (kg) & $63.6(9.1)$ & $62.6(11.5)$ & 0.674 & $63.9(13.0)$ & $61.9(9.0)$ & 0.378 \\
\hline $\operatorname{BMI}\left(\mathrm{kg} / \mathrm{m}^{2}\right)$ & $23.6(3.3)$ & $23.0(3.2)$ & 0.450 & $23.5(3.4)$ & $22.8(3.1)$ & 0.298 \\
\hline Preoperative LVEF (\%) & $63.0(61.0-65.0)$ & $63.0(60.0-66.0)$ & 0.779 & $62.0(58.8-65.0)$ & $65.0(60.0-67.5)$ & 0.077 \\
\hline Clinical manifestations & $15(62.5)$ & $53(66.3)$ & 0.925 & $28(59.6)$ & $40(70.2)$ & 0.356 \\
\hline \multicolumn{7}{|l|}{ Comorbidity } \\
\hline Stroke & $1(4.2)$ & $5(6.3)$ & 1.000 & $2(4.3)$ & $4(7.0)$ & 0.687 \\
\hline Dysarrhythmia & $1(4.2)$ & $7(8.8)$ & 0.678 & $6(12.8)$ & $2(3.5)$ & 0.136 \\
\hline Hyperlipidemia & $1(4.2)$ & $4(5.0)$ & 1.000 & $2(4.3)$ & $3(5.3)$ & 1.000 \\
\hline Diabetes mellitus & $7(29.2)$ & $27(33.8)$ & 0.864 & $17(36.2)$ & $17(29.8)$ & 0.634 \\
\hline \multicolumn{7}{|l|}{ Preoperative CT findings } \\
\hline HU on unenhanced CT & $30.9(5.7)$ & $35.0(8.7)$ & 0.055 & $31.5(7.9)$ & $36.3(7.9)$ & 0.009 \\
\hline Tumor diameter $(\mathrm{cm})$ & $5.0(2.8-45.7)$ & $5.6(3.8-13.5)$ & 0.852 & $8.0(4.0-36.0)$ & $4.8(3.4-8.0)$ & 0.050 \\
\hline \multicolumn{7}{|l|}{ Preoperative catecholamine levels } \\
\hline Plasma norepinephrine (pg/ml) & $350.6(121.3-749.8)$ & $338.8(2.0-1004.7)$ & 0.866 & $358.0(128.0-946.0)$ & $326.3(0.8-897.0)$ & 0.364 \\
\hline Plasma epinephrine $(\mathrm{pg} / \mathrm{ml})$ & $35.4(18.8-181.6)$ & $40.4(0.4-154.5)$ & 0.679 & $40.0(10.6-164.8)$ & $36.1(0.5-172.1)$ & 0.863 \\
\hline Urine vanillylmandelic acid (mg/day) & $9.9(5.2-20.4)$ & $9.5(6.6-20.5)$ & 0.609 & $9.2(6.4-18.7)$ & $10.4(5.5-21.9)$ & 0.847 \\
\hline Urine metanephrine ( $\mu \mathrm{g} /$ day $)$ & $1662.3(866.7-6655.3)$ & $3100.5(1099.4-6705.0)$ & 0.433 & $2585.0(953.8-4954.8)$ & $3686.0(930.0-8303.2)$ & 0.317 \\
\hline \multicolumn{7}{|l|}{ Preoperative MBP (mmHg) } \\
\hline Before premedication & $96.7(88.3-105.0)$ & $100.0(93.3-111.7)$ & 0.186 & $100.0(92.3-109.9)$ & $98.5(93.3-113.7)$ & 0.608 \\
\hline Before induction of anesthesia & $88.4(85.4-93.3)$ & $106.3(97.8-117.5)$ & $<0.001$ & $106.3(90.0-110.3)$ & $95.0(86.7-117.5)$ & 0.311 \\
\hline \multicolumn{7}{|l|}{ Premedication } \\
\hline a-blocker & $19(79.2)$ & $74(92.5)$ & 0.121 & 44 (93.6) & $49(86.0)$ & 0.337 \\
\hline Duration of pretreatment (days) & $25.50(9.25-40.00)$ & $30.00(21.00-46.00)$ & 0.137 & $32.0(23.8-50.0)$ & $27.0(11.0-42.0)$ & 0.063 \\
\hline
\end{tabular}




\begin{tabular}{|c|c|c|c|c|c|c|}
\hline \multirow[t]{2}{*}{ Variables } & \multicolumn{3}{|c|}{ Intraoperative hypotension } & \multicolumn{3}{|l|}{ Hypertensive crisis } \\
\hline & No. $(n=24)$ & Yes $(n=80)$ & $P$ value & No. $(n=47)$ & Yes $(\mathrm{n}=57)$ & P value \\
\hline \multicolumn{7}{|l|}{ Intraoperative data } \\
\hline Induction agent & & & 0.083 & & & 0.592 \\
\hline Thiopental & $10(41.7)$ & $23(28.7)$ & & $16(34.0)$ & $17(29.8)$ & \\
\hline Propofol & $13(54.2)$ & $57(71.3)$ & & $30(63.8)$ & $40(70.2)$ & \\
\hline Etomidate & $1(4.2)$ & $0(0.0)$ & & $1(2.1)$ & $0(0.0)$ & \\
\hline Maintenance agent & & & 1.000 & & & 1.000 \\
\hline Inhalation & $23(95.8)$ & $78(97.5)$ & & $46(97.9)$ & $55(96.5)$ & \\
\hline Propofol & $1(4.2)$ & $2(2.5)$ & & $1(2.1)$ & $2(3.5)$ & \\
\hline Remifentanil & $18(75.0)$ & $59(73.8)$ & 1.000 & $35(74.5)$ & $42(73.7)$ & 1.000 \\
\hline Anesthetic technique & & & 0.008 & & & 0.542 \\
\hline General anesthesia alone & $18(75.0)$ & $33(41.3)$ & & $21(44.7)$ & $30(52.6)$ & \\
\hline Combined epidural-general & $6(25.0)$ & $47(58.8)$ & & $26(55.3)$ & $27(47.4)$ & \\
\hline Methods of surgery & & & 1.000 & & & 0.412 \\
\hline Open & $5(20.8)$ & $23(28.7)$ & & 15 (31.9) & $13(22.8)$ & \\
\hline Laparoscopic & $19(79.2)$ & $57(71.3)$ & & $32(68.1)$ & $44(77.2)$ & \\
\hline Operation time (min) & $212.5(168.8-296.3)$ & $195.0(150.0-247.5)$ & 0.324 & $195.0(150.0-240.0)$ & $210.0(165.0-270.0)$ & 0.294 \\
\hline Amount of fluid administration (ml) & $1900.0(1400.0-3300.0)$ & $2300.0(1800.0-3800.0)$ & 0.143 & $2200(1600-2900)$ & $2200(1700-4100)$ & 0.311 \\
\hline $\mathrm{EBL}(\mathrm{ml})$ & $300.0(100.0-500.0)$ & $300.0(100.0-500.0)$ & 0.768 & $200.0(100.0-500.0)$ & $300.0(100.0-500.0)$ & 0.315 \\
\hline Urine output (ml) & $300.0(150.0-800.0)$ & $400.0(200.00-900.000)$ & 0.247 & $400.0(200.0-630.0)$ & $400.0(200.0-1100.0)$ & 0.926 \\
\hline Transfusion & $2(8.3)$ & $20(25.0)$ & 0.142 & $8(17.0)$ & $14(24.6)$ & 0.487 \\
\hline Hypertensive crisis & $15(62.5)$ & $42(52.5)$ & 0.529 & $38(80.9)$ & $42(73.7)$ & 0.529 \\
\hline
\end{tabular}

Data were presented as mean (SD), median (IQR), and absolute numbers (\%). American society of anesthesiologists (ASA), body mass index (BMI), left ventricular ejection fraction (LVEF), computed tomography (CT), Hounsfield unit (HU), mean blood pressure (MBP).

Table 3. Univariate analysis of variables associated with intraoperative hypotension and hypertensive crisis

\begin{tabular}{|c|c|c|c|c|}
\hline \multirow[t]{2}{*}{ Variable } & \multicolumn{2}{|l|}{ Intraoperative hypotension } & \multicolumn{2}{|l|}{ Hypertensive crisis } \\
\hline & Unadjusted OR (95\% CI) & P value & Unadjusted OR (95\% CI) & P value \\
\hline Age (yr) & $1.000(0.967-1.034)$ & 0.998 & $0.966(0.938-0.996)$ & 0.025 \\
\hline Female gender (Ref. Male) & $1.017(0.407-2.540)$ & 0.971 & $0.953(0.439-2.068)$ & 0.903 \\
\hline ASA classification & $1.265(0.488-3.282)$ & 0.628 & $0.597(0.260-1.370)$ & 0.224 \\
\hline BMI $\left(\mathrm{kg} / \mathrm{m}^{2}\right)$ & $0.945(0.817-1.093)$ & 0.446 & $0.936(0.827-1.060)$ & 0.297 \\
\hline Preoperative LVEF (\%) & $0.982(0.908-1.062)$ & 0.649 & $1.038(0.976-1.104)$ & 0.230 \\
\hline Clinical manifestations (Ref. No) & $1.178(0.457-3.038)$ & 0.735 & $1.597(0.708-3.601)$ & 0.259 \\
\hline \multicolumn{5}{|l|}{ Comorbidity } \\
\hline Stroke (Ref. No) & $1.533(0.170-13.801)$ & 0.703 & $1.698(0.297-9.706)$ & 0.552 \\
\hline Dysarrhythmia (Ref. No) & $2.205(0.258-18.878)$ & 0.470 & $0.248(0.048-1.295)$ & 0.098 \\
\hline Hyperlipidemia (Ref. No) & $1.211(0.129-11.375)$ & 0.867 & $1.250(0.200-7.811)$ & 0.811 \\
\hline Diabetes mellitus (Ref. No) & $1.237(0.458-3.345)$ & 0.675 & $0.750(0.330-1.707)$ & 0.493 \\
\hline \multicolumn{5}{|l|}{ Preoperative CT findings } \\
\hline $\mathrm{HU}$ on unenhanced CT & $1.068(0.997-1.144)$ & 0.059 & $1.081(1.017-1.149)$ & 0.012 \\
\hline Tumor diameter $(\mathrm{cm})$ & $0.992(0.974-1.009)$ & 0.344 & $0.988(0.972-1.005)$ & 0.168 \\
\hline \multicolumn{5}{|l|}{ Preoperative catecholamine levels } \\
\hline Plasma norepinephrine $(\mathrm{pg} / \mathrm{ml})$ & $1.000(1.000-1.000)$ & 0.524 & $1.000(1.000-1.000)$ & 0.345 \\
\hline Plasma epinephrine $(\mathrm{pg} / \mathrm{ml})$ & $1.001(0.999-1.003)$ & 0.473 & $1.000(1.000-1.000)$ & 0.486 \\
\hline Urine vanillylmandelic acid (mg/day) & $1.007(0.961-1.054)$ & 0.773 & $1.012(0.976-1.050)$ & 0.506 \\
\hline Urine metanephrine $(\mu \mathrm{g} /$ day $)$ & $1.000(1.000-1.000)$ & 0.604 & $1.000(1.000-1.000)$ & 0.563 \\
\hline \multicolumn{5}{|l|}{ Preoperative MBP (mmHg) } \\
\hline Before premedication & $1.024(0.995-1.054)$ & 0.110 & $1.013(0.991-1.035)$ & 0.265 \\
\hline Before induction of anesthesia & $1.073(1.029-1.118)$ & 0.001 & $0.991(0.968-1.014)$ & 0.420 \\
\hline \multicolumn{5}{|l|}{ Premedication } \\
\hline a-blocker (Ref. No) & $3.246(0.894-11.784)$ & 0.074 & $0.418(0.104-1.673)$ & 0.218 \\
\hline Duration of pretreatment (day) & $1.012(0.992-1.033)$ & 0.233 & $0.999(0.995-1.003)$ & 0.551 \\
\hline \multicolumn{5}{|l|}{ Intraoperative data } \\
\hline Open surgery (Ref. laparoscopic) & $1.533(0.512-4.596)$ & 0.445 & $0.630(0.264-1.506)$ & 0.299 \\
\hline Operation time (min) & $0.998(0.994-1.002)$ & 0.305 & $1.002(0.998-1.005)$ & 0.370 \\
\hline Inhalation agent (Ref. Propofol) & $0.590(0.051-6.801)$ & 0.672 & $1.673(0.147-19.042)$ & 0.678 \\
\hline Remifentanil (Ref. No) & $0.937(0.328-2.675)$ & 0.903 & $0.960(0.398-2.318)$ & 0.928 \\
\hline GE technique (Ref. GA technique) & $4.273(1.532-11.915)$ & 0.006 & $0.727(0.335-1.578)$ & 0.420 \\
\hline Hypertensive crisis (Ref. No) & $0.663(0.260-1.690)$ & 0.390 & & \\
\hline Intraoperative hypotension (Ref. No) & & & $0.663(0.260-1.690)$ & 0.390 \\
\hline
\end{tabular}


Table 4. Multivariate analysis of variables associated with intraoperative hypotension and hypertensive crisis

\begin{tabular}{|c|c|c|}
\hline Variable & Adjusted OR* $(95 \% \mathrm{CI})$ & $P$ value \\
\hline \multicolumn{3}{|l|}{ Intraoperative hypotension } \\
\hline HU on unenhanced CT & $1.112(1.009-1.226)$ & 0.033 \\
\hline $\mathrm{MBPb}(\mathrm{mmHg})$ & $1.063(1.012-1.117)$ & 0.015 \\
\hline a-blocker (Ref. No) & $5.459(0.937-31.805)$ & 0.059 \\
\hline GE techniques (Ref. GA technique) & $5.439(1.410-20.977)$ & 0.014 \\
\hline \multicolumn{3}{|l|}{ Hypertensive crisis } \\
\hline Dysarrhythmia (Ref. No) & $0.206(0.032-1.318)$ & 0.095 \\
\hline HU on unenhanced CT & $1.087(1.021-1.158)$ & 0.009 \\
\hline GE techniques (Ref. GA technique) & $0.378(0.141-1.015)$ & 0.053 \\
\hline
\end{tabular}

\section{Discussion}

In this retrospective chart review study, intraoperative hypotension was observed in 80 $(76.9 \%)$ patients during PPGL resection. The independent predictors of intraoperative hypotension were an increased $\mathrm{HU}$ value on unenhanced $\mathrm{CT}$, a high $\mathrm{MBPb}$, and the GE technique. Hypertensive crisis occurred in $57(54.8 \%)$ patients during PPGL resection. The only independent predictor of a hypertensive crisis was an increased $\mathrm{HU}$ value on unenhanced CT.

The known risk factors for hemodynamic instability during PPGL surgery are increased preoperative urinary catecholamine levels, larger tumor size, and absence of the a-blocker premedication $[1,8,13,16]$. Previous studies have demonstrated the usefulness of the HU value on unenhanced CT as a diagnostic tool for PPGLs [17]. However, to date, no studies have been conducted on the associations between intraoperative hemodynamic instability during PPGLs surgery and HU value on CT scans. In the present study, an increased HU value on unenhanced CT was found to be associated with the occurrence of both intraoperative hypotension and hypertensive crisis during surgical resection of PPGL. The underlying mechanism of this association is elucidative; however, several studies showed that the increased urinary MN or normetanephrine significantly associated with an increased CT attenuation in PPGLs $[18,19]$.

In the present study, a high $\mathrm{MBPb}$ was found to be associated with the occurrence of intraoperative hypotension, but not with hypertensive crisis during surgery. Before the resection of PPGLs, blood pressure should be optimized whenever possible, since uncontrolled preoperative hypertension in PPGL patients is known to increase the risk of perioperative complications [20]. PPGLs can induce and exacerbate arterial stiffness, myocardial hypertrophy and fibrosis, and these cardiovascular remodeling have been reported to be associated with high blood pressure [21-24]. These hypertensionassociated morphofunctional changes impair the ability of blood pressure regulation in stressful situations $[25,26]$, which is thought to contribute to the development of intraoperative hypotension during PPGL resection. According to the current recommendations based on recent research, preoperative blood pressure control for PPGL patients is strict; blood pressure should be below 130/85 mmHg (MBP $100 \mathrm{mmHg}$ ) in the seated position, while SBP should exceed $90 \mathrm{mmHg}$ in the standing position [5]. In the present study, the cut-off value of $\mathrm{MBPb}$ to predict intraoperative hypotension was $93.7 \mathrm{mmHg}$, which is consistent with the aforementioned recommendation.

In the present study, the GE technique was found to be associated with the occurrence of intraoperative hypotension during PPGL surgery. Epidural analgesia is frequently used in combination with general anesthesia in PPGL surgery for the attenuation of intraoperative hemodynamic response and postoperative pain control. Luo et al. [6] demonstrated that, as compared to the GA technique for PPGL resection, the GE technique has a vasodilator-sparing effect by reducing systemic vascular resistance before tumor removal. Furthermore, Li et al. [15] reported that although the GE technique failed to reduce the occurrence of intraoperative hemodynamic fluctuations, it effectively reduced postoperative complications in patients undergoing open surgery for pheochromocytoma. Our results suggest that the GE technique was not effective in preventing hypertensive crisis during PPGL surgery, but it may have exacerbated intraoperative hypotension. This may be due to the fact that the epidural block was not sufficient to inhibit the catecholamine surge during tumor manipulation but exaggerated the sympathectomy and vasodilatory effects after tumor ligation $[2,5,27]$.

Tumor manipulation is the most powerful factor to induce a catecholamine surge during PPGL surgery $[12,14,28]$. Other risk factors previously found to be potentially associated with hypertensive crises include increased preoperative catecholamine level, larger tumor size, and absence of a-blocker premedication $[1,5,8,16]$. However, in the present study, these factors were not found to be associated with hypertensive crisis during PPGL surgery. This controversial outcome may be due to the most powerful factor, surgical manipulation of PPGL, masking the effects of other factors on hypertensive crisis. The surgeon's skill and competence levels are expected to have a significant impact on minimizing tumor manipulation; however, we could not evaluate 
the impact of this factor, which is a limitation of the present study.

The present study has several other limitations. First, the intraoperative hemodynamic data used in the present study were extracted from electronic anesthetic records. Since these measurements are recorded every 5 minutes, the accuracy of the hemodynamic parameters could have been compromised. Second, due to the low prevalence rate of PPGLs, the recruitment period was over 18 years, and the development of surgical and anesthetic skills may have produced a bias. Finally, due to the low frequency of complications, we could not analyze the correlation between intraoperative hemodynamic instability and the patient's clinical outcome.

\section{Conclusions}

In conclusion, in the present study, an increased $\mathrm{HU}$ value on unenhanced $\mathrm{CT}$, a high $\mathrm{MBPb}$, and the GE anesthetic technique were found to be independent predictors of intraoperative hypotension, while an increased $\mathrm{HU}$ value on unenhanced CT was found to be an independent predictor of hypertensive crisis during PPGL surgery. Since the GE anesthetic technique has the potential to cause intraoperative hypotension, anesthesiologists should consider these findings before selecting an anesthetic technique in PPGL surgery.

\section{Abbreviations}

95\% CI: 95\% confidence interval; ASA: American Society of Anesthesiologists; AUROC: Area under the receiver operating characteristic curve; CT: Computed tomography; GA: General anesthesia alone; GE: Combined epidural-general anesthesia technique; HU: Hounsfield unit; IQR: Interquartile range; MBPpre: Mean blood pressure before premedication; $\mathrm{MBPb}$ : Mean blood pressure immediately before induction of anesthesia; OR: Odds ratio; pEpi: Plasma epinephrine; pNE: Plasma norepinephrine; PPGLs: Pheochromocytomas and sympathetic paragangliomas; SBP: Systolic blood pressure; SBPb: Systolic blood pressure immediately before induction of anesthesia; SD: Standard deviation; uMN: Urine metanephrine; uVMA: Urine vanillylmandelic acid.

\section{Competing Interests}

The authors have declared that no competing interest exists.

\section{References}

1. Lenders JW, Duh QY, Eisenhofer G, et al. Pheochromocytoma and paraganglioma: An endocrine society clinical practice guideline. J Clin Endocrinol Metab. 2014; 99: 1915-42.

2. Bajwa SS, Bajwa SK. Implications and considerations during pheochromocytoma resection: A challenge to the anesthesiologist. Indian J Endocrinol Metab. 2011; 15 (Suppl 4): S337-S344.
3. Berends AMA, Buitenwerf $E$, de Krijger RR, et al. Incidence of pheochromocytoma and sympathetic paraganglioma in the Netherlands: A nationwide study and systematic review. Eur J Intern Med. 2018; 51: 68-73.

4. Ramachandran R, Rewari V. Current perioperative management of pheochromocytomas. Indian J Urol. 2017; 33: 19-25.

5. Wang H, Jepegnanam C. Recognition and management of pheochromocytoma and paraganglioma. Anaesthesia and Intensive Care Medicine. 2017; 18: 496501.

6. Luo A, Guo X, Yi J, et al. Clinical features of pheochromocytoma and perioperative anesthetic management. Chin Med J. 2003; 116: 1527-31.

7. Woodrum DT, Kheterpal S. Anesthetic management of pheochromocytoma. World Journal of Endocrine Surgery. 2010; 2: 111-7.

8. Kwon SY, Lee KS, Lee JN, et al. Risk factors for hypertensive attack during pheochromocytoma resection. Investig Clin Urol. 2016; 57: 184-90.

9. Agrawal R, Mishra SK, Bhatia E, et al. Prospective study to compare peri-operative hemodynamic alterations following preparation for pheochromocytoma surgery by phenoxybenzamine or prazosin. World J Surg. 2014; 38: 716-23.

10. Kinney MA, Warner ME, vanHeerden JA, et al. Perianesthetic risks and outcomes of pheochromocytoma and paraganglioma resection. Anesth Analg. 2000; 91: 1118-23.

11. Ramakrishna H. Pheochromocytoma resection: Current concepts in anesthetic management. J Anaesthesiol Clin Pharmacol. 2015; 31: 317-23.

12. Joris JL, Hamoir EE, Hartstein GM, et al. Hemodynamic changes and catecholamine release during laparoscopic adrenalectomy for pheochromocytoma. Anesth Analg. 1999; 88: 16-21.

13. Wu S, Chen $\mathrm{W}$, Shen $\mathrm{L}$, et al. Risk factors for prolonged hypotension in patients with pheochromocytoma undergoing laparoscopic adrenalectomy: A single-center retrospective study. Sci Rep. 2017; 7: 5897.

14. Connor D, Boumphrey S. Perioperative care of phaeochromocytoma. BJA Education. 2015; 16: 153-8.

15. Li N, Kong H, Li SL, et al. Combined epidural-general anesthesia was associated with lower risk of postoperative complications in patients undergoing open abdominal surgery for pheochromocytoma: A retrospective cohort study. PLoS One. 2018; 13: e0192924.

16. Namekawa T, Utsumi T, Kawamura K, et al. Clinical predictors of prolonged postresection hypotension after laparoscopic adrenalectomy for pheochromocytoma. Surgery. 2016; 159: 763-70.

17. Buitenwerf E, Korteweg T, Visser A, et al. Unenhanced CT imaging is highly sensitive to exclude pheochromocytoma: a multicenter study. Eur J Endocrinol. 2018; 178: 431-7.

18. Kannan S, Purysko A, Faiman C, et al. Biochemical and radiological relationships in patients with pheochromocytoma: Lessons from a case control study. Clin Endocrinol. 2014; 80: 790-6.

19. Sane T, Schalin-Jäntti C, Raade M. Is biochemical screening for pheochromocytoma in adrenal incidentalomas expressing low unenhanced attenuation on computed tomography necessary? J Clin Endocrinol Metab. 2012; 97: 2077-83.

20. Plouin PF, Duclos JM, Soppelsa F, et al. Factors associated with perioperative morbidity and mortality in patients with pheochromocytoma: Analysis of 165 operations at a single center. J Clin Endocrinol Metab. 2001; 86: 1480-6.

21. Petrák O, Strauch B, Zelinka T, et al. Factors influencing arterial stiffness in pheochromocytoma and effect of adrenalectomy. Hypertens Res. 2010; 33: 454-9.

22. Zuber SM, Kantorovich V, Pacak K. Hypertension in pheochromocytoma: Characteristics and treatment. Endocrinol Metab Clin North Am. 2011; 40: 295-311.

23. Galetta F, Bernini G, Franzoni F, et al. Preclinical cardiac involvement in phaeochromocytoma: A study with integrated backscatter. Clin Endocrinol. 2008; 68: 756-61.

24. Tomek J, Bub G. Hypertension-induced remodelling: on the interactions of cardiac risk factors. J Physiol. 2017; 595: 4027-36.

25. Alecu C, Cuignet-Royer E, Mertes PM, et al. Pre-existing arterial stiffness can predict hypotension during induction of anaesthesia in the elderly. Br J Anaesth. 2010; 105: 583-8.

26. Lim PO, MacFadyen RJ, Clarkson PB, et al. Impaired Exercise Tolerance in Hypertensive Patients. Ann Intern Med. 1996; 124: 41-55.

27. Roizen MF, Horrigan RW, Koike M, et al. A prospective randomized trial of four anesthetic techniques for resection of pheochromocytoma. Anesthesiology. 1982; 57: a43

28. Lentschener C, Gaujoux S, Tesniere A, et al. Point of controversy: Perioperative care of patients undergoing pheochromocytoma removal-time for a reappraisal? Eur J Endocrinol. 2011; 165: 365-73. 